\title{
ENFERMEDAD POR REFLUJO GASTROESOFÁGICO COMO FACTOR ASOCIADO A ASMA BRONQUIAL EN POBLACIÓN PEDIÁTRICA
}

\author{
GASTROESOPHAGEAL REFLUX DISEASE AS A FACTOR ASSOCIATED WITH BRONCHIAL ASTHMA \\ IN THE PEDIATRIC POPULATION \\ Rafael Ricra', Juan Carlos Roque², Joseph Alburqueque-Melgarejo ${ }^{3}$ \\ Pedro Ricra ${ }^{4}$, Claudia Saldaña ${ }^{5}$
}

\begin{abstract}
RESUMEN
Objetivo: Determinar la relación entre enfermedad por reflujo gastroesofágico y asma bronquial en población pediátrica en el hospital Nacional del Niño entre los años 2017-2018. Métodos: Se realizó un estudio observacional analítico tipo casos y controles no emparejado de razón 1 caso para 2 controles, con un total de 45 casos y 90 controles, se realizó un muestreo probabilístico aleatorio simple, con una recolección retrospectiva para las variables asma bronquial, ERGE, esofagitis inducida por ERGE, prematuridad, lactancia materna interrumpida, sexo y edad. Se realizó un modelo de regresión logística bivariado y múltiple para la obtención del Odds Ratio para la fuerza de asociación. Resultados: Se encontró una diferencia estadísticamente significativa para ERGE $(p=0,002)$, siendo 33,33\% y $11,11 \%$ para los casos y controles, así como para lactancia interrumpida $(\mathrm{p}=0,013)$; por análisis de regresión logística múltiple las variables ERGE (OR 4,27, $p=0,003$, IC 1,64-10,92) y lactancia materna interrumpida (OR 2,74, $\mathrm{P}=0,011$, IC 1,26-5,97) presentaron relación para asma bronquial. Conclusión: Se encontró una relación entre ERGE y asma bronquial la cual puede ser extrapolada a la población pediátrica de la ciudad de Lima, este es el primer artículo publicado al respecto a nivel nacional, se recomiendan estudios prospectivos para determinar causalidad.
\end{abstract}

Palabras clave: Enfermedad por reflujo gastroesofágico; Asma bronquial; Pediatría (fuente: DeCS BIREME).

\begin{abstract}
Objective: To determine the relationship between gastroesophageal reflux disease and bronchial asthma in the pediatric population of the National Children's Hospital Breña headquarters between the years 2017-2018. Methods: An analytical observational study of cases and controls was not matched, with a ratio of 1 case to 2 controls, with a total of 45 cases and 90 controls, a simple random probability sampling was performed, with a retrospective collection for the variables bronchial asthma, GERD, GERD-induced esophagitis, prematurity, interrupted breastfeeding, sex and age. A bivariate and multiple logistic regression model was performed to obtain the Odds Ratio for the strength of association. Results: A statistically significant difference was found for GERD ( $p=0.002$ ), being $33.33 \%$ and $11.11 \%$ for cases and controls, as well as for interrupted lactation ( $p$ $=0.013$ ); by multiple logistic regression analysis the variables GERD (OR 4.27, $p=0.003, \mathrm{Cl} 1.64-10.92)$ and interrupted breastfeeding (OR 2.74, $\mathrm{P}=0.011, \mathrm{Cl} 1.26-5.97$ ) presented a relationship for bronchial asthma. Conclusion: A relationship was found between GERD and bronchial asthma which can be extrapolated to the pediatric population of the city of Lima, this is the first article published in this regard at the national level, prospective studies are recommended to determine causality.
\end{abstract}

Key words: Gastroesophageal reflux disease; Bronchial asthma; Pediatrics (source: MeSH NLM).

${ }^{1}$ Universidad Privada San Juan Bautista, Lima-Perú.

${ }^{2}$ Universidad Científica del Sur, Lima-Perú.

${ }^{3}$ Universidad Ricardo Palma, Lima-Perú.

4 Instituto Nacional del Niño sede Breña, Lima-Perú.

5 Instituto Nacional Materno Perinatal, Lima-Perú.

Citar como: Rafael Ricra, Juan Carlos Roque, Joseph Alburqueque-Melgarejo, Pedro Ricra, Claudia Saldaña. Enfermedad por reflujo gastroesofágico como factor asociado a asma bronquial en población pediátrica Rev. Fac. Med. Hum. Abril 2020; 20(2):281-286. DOI 10.25176/RFMH.v20i2.2918 


\section{INTRODUCCIÓN}

La enfermedad por reflujo gastroesofágico (ERGE) se define como flujo de contenido gástrico al esófago asociado a síntomas y/o complicaciones que pueden ser de tipo esofágicas o extra esofágicas. El ERGE representa una patología gastrointestinal frecuente alrededor del globo con prevalencias estimadas entre 10-20\% en América y Europa; y 5\% en Asia ${ }^{(1,2,3,4)}$.

Actualmente, su diagnóstico se ha incrementado particularmente en la población pediátrica, donde se presenta más frecuentemente en el sexo masculino. El ERGE puede presentarse de manera fisiológica hasta antes del primer año de vida por inmadurez de la unión gastroesofágica, condición que disminuye luego del primer año de vida ${ }^{(3)}$.

Suprevalenciaes mayor en niños que sufren deobesidad, enfermedades neurológicas, enfermedades cardiacas congénitas, anormalidades del tracto gastrointestinal, hernia diafragmática congénita y anormalidades cromosómicas. Los nacidos prematuros también tienen riesgo de desarrollar ERGE por inmadurez del esfínter esofágico inferior, alteraciones en la peristalsis esofágica, alta ingesta de leche, y vaciamiento gástrico retardado ${ }^{(3,4)}$. Por otra parte, la lactancia materna exclusiva representa un factor protector para el ERGE ${ }^{(3)}$.

Las complicaciones respiratorias del ERGE son frecuentes en la población pediátrica, siendo el asma la más ampliamente estudiada y cuya ocurrencia se atribuye principalmente a mecanismos vagales, inflamación neurogénica, microaspiración de ácido y reactividad bronquial incrementada $a^{(5,6,7,8,9)}$.

En los últimos años, múltiples estudios han demostrado la existencia de una relación entre asma y ERGE. Una revisión sistemática que revisó 18 artículos indicó una prevalencia promedio de $23,4 \%$ de ERGE en pacientes con asma ${ }^{(10)}$. Otra revisión sistemática que revisó un total de 20 artículos encontró una prevalencia de $22,0 \%$ de ERGE en pacientes con asma con un OR acumulado de 5,6 (IC:95\%: 4,3-6,9) $)^{(11)}$. Sin embargo, muchos de estos estudios indican una relación de tipo bidireccional entre estas dos variables ${ }^{(10,11,12,13)}$. Por lo que suponemos una asociación entre estas variables y presentamos el siguiente estudio con el objetivo de determinar la asociación entre la enfermedad de reflujo gastroesofágico (ERGE) y el asma bronquial en población pediátrica del Instituto Nacional de Salud del Niño durante el periodo 2017-2018. Este es el primer trabajo a nivel nacional en mencionar esta asociación.

\section{MÉTODOS}

\section{Diseño}

Se realizó un diseño de estudio tipo observacional, analítico, retrospectivo, casos y controles no emparejado de una proporción de 1 caso para 2 controles. El presente estudio se realizó en el instituto de nacional del niño sede breña en el año 2018 donde se realizó una recolección de datos de pacientes atendidos entre los años 2017-2018. La población objetivo fueron aquellos pacientes pediátricos con edades superiores a 5 años con manifestaciones clínicas de las vías respiratorias atendidas en consulta externa del servicio de pediatría general, donde los casos fueron aquellos pacientes con asma bronquial y los controles aquellos pacientes sin asma bronquial. Como factor de exposición se tomó al reflujo gastroesofágico diagnosticado por $\mathrm{PH}$-metria esofágica.

\section{Procedimientos y variables}

Dentro de los criterios de selección para los casos y controles se tomaron como criterios de inclusión prueba de $\mathrm{PH}$-metria, endoscopia alta, historia clínica con datos completos; como criterios se exclusión se tomaron parálisis cerebral infantil, anomalías orgánicas de la vía respiratoria y digestiva superior, instrumentos invasivos a las vías respiratorias y digestivas, neoplasias y patologías reumáticas.

Las variables independientes en el estudio fueron la ERGE, cuyo instrumento diagnóstico fue la $\mathrm{PH}$-metria y la esofagitis cuyo instrumento diagnóstico fue la biopsia obtenida por endoscopia digestiva alta, las cuales fueron tomadas y evaluadas por especialistas de gastroenterología y patología respectivamente. La variable dependiente, asma bronquial empleo como instrumento diagnostico al espirómetro de acuerdo a las recomendaciones de la guía $\operatorname{GINA}^{(14)}$. El cual fue dado por especialistas de consulta externa de pediatría. Como otras variables se registró la edad en años del paciente, género, prematuridad y lactancia materna interrumpida y parto prematuro, las cuales fueron tomadas de la anamnesis y antecedentes presentes en la fuente de información secundaria, la historia clínica.

Los datos de cada una de las variables mencionadas fueron registrados en una ficha de recolección de datos para cada sujeto de estudio las cuales se almacenaron para su posterior vaciado y análisis estadístico.

\section{Población y muestra}

Para el cálculo del tamaño muestral se tomó un intervalo de confianza del $95 \%$, una potencia estadística del $80 \%$, una proporción de 1:2 para casos y controles 
respectivamente, tomaron los resultados obtenidos por Debley et al. ${ }^{(15)}$ con una proporción de casos expuestos de $19,3 \%$, proporción de controles expuestos 2,5\% y un Odds ratio de 9,2, se empleó el paquete epidemiológico OpenEpi de acceso libre para el cálculo muestral donde se obtuvo un total de 135 pacientes, siendo 45 casos y 135 controles respectivamente empleando la fórmula de Fleiss con corrección de continuidad. Se realizó un muestreo de tipo probabilístico unietapico donde se estratifico a la población en pacientes con asma bronquial y sin asma bronquial, se realizó un muestreo aleatorio simple en cada uno de los estratos, sin reposición.

\section{Cuestiones éticas}

El presente estudio respeto el anonimato de cada uno de los sujetos de estudio no se revelaron nombres ni documentos de identidad, se contó con la aprobación para la ejecución por parte de las autoridades de la institución.

\section{Análisis estadístico}

Las fichas de recolección de datos fueron vaciadas a una hoja de cálculo Excel, donde por medio del programa Stat-Tranfer, fueron transportados al paquete estadístico STATA versión 15 para su posterior análisis estadístico

Los estadísticos descriptivos se presentan para los casos y los controles reportándose la media y desviación estándar para la variable cuantitativa edad, así como la frecuencia absoluta y relativa para cada variable cualitativa, esto es sexo, esofagitis y lactancia materna interrumpida.

Para la estadística inferencial se realizó la prueba estadística no paramétrica chi cuadrado para contrastar las variables cualitativas y una prueba de contraste de medias para las variables cuantitativas respecto a los casos y controles, posteriormente se realizó un modelo de regresión logística bivariado, para la obtención del Odds ratio e intervalos de confianza al 95\%. Para concluir se ejecuta una modelo de regresión logística multivariado para la obtención de un Odds ratio ajustado con sus respectivos intervalos de confianza al $95 \%$, con aquellas variables que son consideradas como factores de riesgo para el asma bronquial.

\section{RESULTADOS}

Del total muestral de 135 pacientes pediátricos ninguno de los pacientes estudiados presentó datos perdidos para las variables estudiadas, 45 fueron pacientes con asma y 90 pacientes no presentaban asma, la media y desviación estándar de edad para ambos grupos fue $10,73+/-1,95$ y 10,79 +/- 1,76 respectivamente, sin presentar diferencias estadísticamente significativas. El porcentaje de sexo masculino fue superior en los pacientes asmáticos $60 \%$, en relación a los pacientes no asmáticos 48,89\%, sin encontrarse diferencia estadísticamente significativa entre los grupos con un $p=0,223$. Elporcentajedelactanciamaternainterrumpida fue inferior en pacientes asmáticos $44,44 \%$, en relación a los pacientes no asmáticos $66,67 \%$, encontrándose una diferencia estadísticamente significativa entre los grupos con un $p=0,013$. El porcentaje de prematuridad fue superior en pacientes asmáticos 15,56\%, en relación a los pacientes no asmáticos 8,89\%, sin encontrarse diferencia estadísticamente significativa entre los grupos con un $p=0,245$. El porcentaje de ERGE fue superior en pacientes asmáticos 33,33\%, en relación a los pacientes no asmáticos $11,11 \%$, encontrándose una diferencia estadísticamente significativa entre los grupos con un $p=0,002$. El porcentaje de esofagitis asociada a ERGE fue superior en pacientes asmáticos $17,78 \%$, en relación a los pacientes no asmáticos $7,78 \%$, sin encontrarse una diferencia estadísticamente significativa entre los grupos con un $p=0,081$ (tabla 1).

Se realizó un modelo de regresión logística bivariado para las variables sexo (OR 1,57, $p=0,244, \mathrm{IC} 0,76-3,24)$ lactancia materna interrumpida (OR 2,5, $P=0,014$, IC 1,20-5,21), prematuridad (OR 1,89, $p=0,251$, IC 0,645,59), ERGE (OR 3,99, $p=0,003, I C 1,62-9,87)$, esofagitis inducida por ERGE (OR 2,56, p=0,089, IC 0,87-7,60), solo las variables ERGE y la lactancia materna interrumpida fueron las únicas variables que presentaron significancia estadística (tabla 2).

Se realizó un modelo de regresión logística múltiple para ajustar el Odds ratio de ERGE por variables que presentan un efecto demostrado sobre el asma bronquial, ERGE (OR 4,27, $p=0,003$, IC 1,64-10,92), sexo (OR 1,61, $p=0,235$, IC 0,74-3,50) lactancia materna interrumpida (OR 2,74, $\mathrm{P}=0,011$, IC 1,26-5,97), prematuridad (OR 1,92, $\mathrm{p}=0,274, \mathrm{IC}$ 0,60-6,18) (tabla 3) las variables ERGE y lactancia materna fueron las únicas que presentaron significancia estadística. 
Tabla 1. Análisis univariado.

\begin{tabular}{lccc|} 
& $\begin{array}{c}\text { Controles } \\
(\mathbf{N}:) \%(\mathbf{n})\end{array}$ & $\begin{array}{c}\text { Casos } \\
(\mathbf{N}:) \%(\mathbf{n})\end{array}$ & Valor $\mathbf{p}$ \\
\hline ERGE & $11,11 \%(10)$ & $33,33 \%(15)$ & 0,002 \\
\hline Lactancia materna interrumpida & $33,33 \%(30)$ & $55,56(25)$ & 0,013 \\
\hline Parto prematuro & $8,89 \%(8)$ & $15,56 \%(7)$ & 0,245 \\
\hline Sexo masculino & $48,89 \%(44)$ & $60 \%(45)$ & 0,223 \\
\hline Esofagitis inducida por ERGE & $7,78 \%(7)$ & $17,78 \%(8)$ & 0,081 \\
\hline
\end{tabular}

Valores p obtenidos de la prueba no paramétrica Chi cuadrado.

Tabla 2. Análisis con regresión logística bivariado.

\begin{tabular}{|lccc|}
\multicolumn{1}{c}{ Variables } & Odds Ratio & P valor & Intervalo de confianza \\
\hline ERGE & 3,99 & 0,003 & $1,62-9,87$ \\
\hline Lactancia materna interrumpida & 2,50 & 0,014 & $1,20-5,21$ \\
\hline Parto prematuro & 1,89 & 0,251 & $0,64-5,59$ \\
\hline Sexo masculino & 1,57 & 0,224 & $0,76-3,24$ \\
\hline Esofagitis inducida por ERGE & 2,56 & 0,089 & $0,87-7,59$ \\
\hline
\end{tabular}

Tabla 3. Análisis con regresión logística múltiple.

\begin{tabular}{lccc}
\multicolumn{1}{c}{ Variables } & Odds Ratio & P valor & Intervalo de confianza \\
\hline ERGE & 4,24 & 0,003 & $1,64-10,93$ \\
\hline Lactancia materna interrumpida & 2,74 & 0,011 & $1,26-5,97$ \\
\hline Parto prematuro & 1,92 & 0,274 & $0,60-6,18$ \\
\hline Sexo masculino & 1,61 & & $0,74-3,50$ \\
\hline
\end{tabular}




\section{DISCUSIÓN}

El presente estudio demostró una relación entre ERGE y asma bronquial en población pediátrica, asimismo se encontró una prevalencia elevada de ERGE en pacientes con asma. Sin embargo, no se encontró una relación estadísticamente significativa entre esofagitis y asma bronquial. Dicho hallazgo que podría explicarse con el hecho de que puede existir sintomatología de ERGE sin la presencia de hallazgos endoscópicos y que la severidad de estos síntomas tampoco se correlaciona con los hallazgos endoscópicos, como lo señalan los estudios de Zuberi BF et al. y Arul P. et al. ${ }^{(16,17)}$. Por otra parte, hay estudios como el de Ashraf $\mathrm{K}$ et al. que sugieren que, si existe una asociación entre estas variables, por lo que nuestro resultado permanece controversial ${ }^{(18)}$.

Éste estudio brinda evidencia sobre la asociación entre ERGE y asma de acuerdo con estudios publicados previamente ${ }^{(8,23,24)}$. Asimismo, revisiones sistemáticas como las de Thakkar K et al demuestran una prevalencia elevada de ERGE en pacientes con asma $(22,0 \%)$ en relación a los controles $(4,8 \%)^{(11)}$. De manera similar estudios como los de Havemann B. et al muestran una asociación estadísticamente significativa con un OR acumulado de 5,5 (IC 95\%: 1.9-15.8) entre las variables ERGE y asma ${ }^{(12)}$.

Existe evidencia fisiopatológica de esta asociación que sostiene que la presencia de reflujo acido puede ingresar al árbol traqueobronquial y alterar su funcionamiento por varios mecanismos, uno de ellos es estimulando reflejos vagales que incrementen la resistencia de la vía aérea, otros se basan en la estimulación de fibras aferentes nociceptivas que a través de la liberación de neuroquininas como sustancia $P$ o neuroquinina $A$ causen broncoespasmo y aumento en la secreción de moco a nivel bronquial. Otro mecanismo propuesto consiste en el aumento de la reactividad bronquial inducido por la presencia de ácido ${ }^{(8,925)}$. A pesar de la existencia de esta relación que postula al ERGE como potencial causa del asma en niños, también hay estudios que ponen en evidencia una relación inversa, sugiriendo una posible relación bidireccional entre estas variables $^{(8,9,21)}$. Finalmente, el presente estudio se puede extrapolar a poblaciones pediátricas a nivel de Lima metropolitana.
El estudio cuenta con limitaciones propias del diseño. Una de ellas radica en el hecho de que se trata de un estudio unicentrico, lo cual afecta su validez externa. Además, se utilizó información que ya había sido generada previamente y la fuente de información disponible no contaba con datos suficientes para poder evaluar otras variables como medidas antropométricas y el estado nutricional de los pacientes de edad pediátrica, siendo la obesidad un factor de riesgo importante tanto para asma como para ERGE ${ }^{(19,20)}$. Tampoco se evaluaron otras variables como el uso de broncodilatadores para el asma en niños que habían sido diagnosticados con ERGE, cuyo uso ha demostrado aumentar el riesgo de desarrollar asma en pacientes con $\operatorname{ERGE}^{(8,21,22,23)}$. Asimismo, nuestro estudio no evaluó la correlación entre la severidad de asma con prevalencia elevada de ERGE ni tampoco el efecto del tratamiento para ERGE en pacientes con asma.

\section{CONCLUSIÓN}

Se concluyó que hay una relación entre las variables ERGE y lactancia materna interrumpida para la variable asma bronquial. Este es el primer estudio a nivel nacional en realizar esta asociación. Se recomienda realizar estudios prospectivos para poder determinar una asociación de riesgo.

Contribuciones de autoría: Los autores participaron en la génesis de la idea, diseño de proyecto, recolección e interpretación de datos, análisis de resultados y preparación del manuscrito del presente trabajo de investigación.

Financiamiento: Autofinanciado.

Conflicto de interés: Los autores declaran no tener conflicto de interés en la publicación de este artículo.

Recibido: 02 febrero 2020

Aprobado: 10 de marzo 2020

Correspondencia: Leonardo Rafael Ricra Fernández.

Dirección: Jr. Agustín de Jáuregui 437 - La Victoria, Lima-Perú.

Teléfono: 931349975

Correo:leonardo_13_92@hotmail.com 


\section{REFERENCIAS BIBLIOGRÁFICAS}

1. Rybak, A., Pesce, M., Thapar, N., \& Borrelli, O. (2017). Gastro-Esophageal Reflux in Children. International Journal of Molecular Sciences, 18(8), 1671. doi:10.3390/ijms18081671

2. Gonzalez Ayerbe, J. I., Hauser, B., Salvatore, S., \& Vandenplas, Y. (2019) Diagnosis and Management of Gastroesophageal Reflux Disease in Infants and Children: from Guidelines to Clinical Practice. Pediatric Gastroenterology, Hepatology \& Nutrition, 22(2), 107. doi:10.5223/pghn.2019.22.2.107

3. Leung AK, Hon KL. Gastroesophageal reflux in children: an updated review. Drugs Context. 2019 Jun 17;8:212591. doi: 10.7573/dic.212591

4. El-Serag, H. B., Sweet, S., Winchester, C. C., \& Dent, J. (2013). Update on the epidemiology of gastro-oesophageal reflux disease: a systematic review. Gut, 63(6), 871-880. doi:10.1136/gutjnl-2012-304269

5. Ozcan C., Erkoçoğlu M., Civelek E., Demirkan H., Kırsaçlıoğlu C.T., Tiryaki H.T. Giniş T., Kocabaş C.N. The relationship between gastro-oesophageal reflux disease and asthma during childhood. Allergol. Immunopathol. 2014;42:109114. doi: 10.1016/j.aller.2012.08.009.

6. Del Rosario J.F. Orenstein S.R. Evaluation and management of gastroesophageal reflux and pulmonary disease. Curr. Opin. Pediatr. 1996;8:209-215. doi: 10.1097/00008480-199606000-00003.

7. 24. Borrelli O., Marabotto C., Mancini V., Aloi M., Macri F., Falconieri P., Lindley K.J., Cucchiara S. Role of gastroesophageal reflux in children with unexplained chronic cough. J. Pediatr. Gastroenterol. Nutr. 2011;53:287-292. doi: 10.1097/ MPG.0b013e318216e1ad.

8. Ewa Toporowska-Kowalska, Krystyna Wasowska-Królikowska, Aneta Krogulska. Asthma and gastroesophageal reflux in children. Med Sci Monit 2002; 8(3): RA64-71

9. Scarupa, M. D., Mori, N., \& Canning, B. J. (2005). Gastroesophageal Reflux Disease in Children with Asthma. Pediatric Drugs, 7(3), 177-186. doi:10.2165/00148581-200507030-00004

10. Tolia, V., Vandenplas, Y. (2009). Systematic review: the extra-oesophagea symptoms of gastro-oesophageal reflux disease in children. Alimentary Pharmacology \& Therapeutics, 29(3), 258-272. doi:10.1111/j.13652036.2008.03879.x

11. Thakkar, K., Boatright, R. O., Gilger, M. A., \& El-Serag, H. B. (2010) Gastroesophageal Reflux and Asthma in Children: A Systematic Review. PEDIATRICS, 125(4), e925-e930. doi:10.1542/peds.2009-2382

12. Havemann, B. D., Henderson, C. A., \& El-Serag, H. B. (2007). The association between gastro-oesophageal reflux disease and asthma: a systematic review. Gut, 56(12), 1654-1664. doi:10.1136/gut.2007.122465

13. Insight into the relationship between gastroesophageal reflux disease and ashtma - Fehrni Ates MD
14. Global Initiative for Asthma. Global Strategy for Asthma Management and Prevention, 2019. https://ginasthma.org/

15. Debley, J. S., Carter, E. R., \& Redding, G. J. (2006). Prevalence and impact of gastroesophageal reflux in adolescents with asthma: A population-based study. Pediatric Pulmonology, 41(5), 475-481. doi:10.1002/ppul.20399

16. Zuberi BF, Faisal N, Quraishy MS, Afsar S, Kazi LA, Kazim E. Correlation between clinical endoscopic and histological findings at esophago-gastric junction in patients of gastroesophageal reflux disease. J Coll Physicians Surg Pak. 2005 Dec;15(12):774-7. DOI: 12.2005/JCPSP.774777

17. Arul $P$, Vinoth $B$, Alexander T, Phansalkar M, Padhi S. Correlation of narrow band imaging endoscopy and histopathology in the diagnosis of nonerosive reflux disease. Saudi Journal of Gastroenterology: Official Journal of the Saudi Gastroenterology Association, 31 Aug 2015, 21(5):330-336. DOI: 10.4103/13193767.164205

18. Ashraf Karbasi, Mohammad Emami Ardestani, Mostafa Ghanei, Ali Amini Harandi. The association between reflux esophagitis and airway hyperreactivity in patients with gastro-esophageal reflux. J Res Med Sci. 2013 Jun 18(6): 473-476

19. Lorenza Di Genova, Laura Penta, Anna Biscarini, Giuseppe Di Cara, Susanna Esposito. Children with Obesity and Asthma: Which Are the Best Options for Their Management? Nutrients. 2018 Nov; 10(11): 1634. doi: 10.3390/ nu10111634

20. Hoda M Malaty, J Kennard Fraley, Suhaib Abudayyeh et al. Obesity and gastroesophageal reflux disease and gastroesophageal reflux symptoms in children. Clin Exp Gastroenterol. 2009; 2: 31-36. doi: 10.2147/ceg.s4715

21. Fehmi Ates, Michael F Vaezi. Insight Into the Relationship Between Gastroesophageal Reflux Disease and Asthma. Gastroenterol Hepatol (N Y). 2014 Nov; 10(11): 729-736.

22. Solana García Maria José, López-Herce Cid Jesús, Sánchez Sánchez César. Gastroesophageal Reflux in Critically III Children: A Review. ISRN Gastroenterol. 2013; 2013: 824320.doi: 10.1155/2013/824320

23. K Stordal, G Johannesdottir, B Bentsen, P Knudsen, K Carlsen et al. Acid suppression does not change respiratory symptoms in children with asthma and gastro-oesophageal reflux disease . Arch Dis Child. 2005 Sep; 90(9): 956960. doi: $10.1136 /$ adc.2004.068890

24. Blake, K., \& Teague, W. G. (2012). Gastroesophageal reflux disease and childhood asthma. Current Opinion in Pulmonary Medicine, 1. doi:10.1097/ mcp.0b013e32835b582b

25. Khoshoo, V., Mohnot, S., Haydel, R., Saturno, E., Edell, D., \& Kobernick, A. (2009). Bronchial hyperreactivity in non-atopic children with asthma and reflux: Effect of anti-reflux treatment. Pediatric Pulmonology, 44(11), 1070 1074. doi:10.1002/ppul.21094

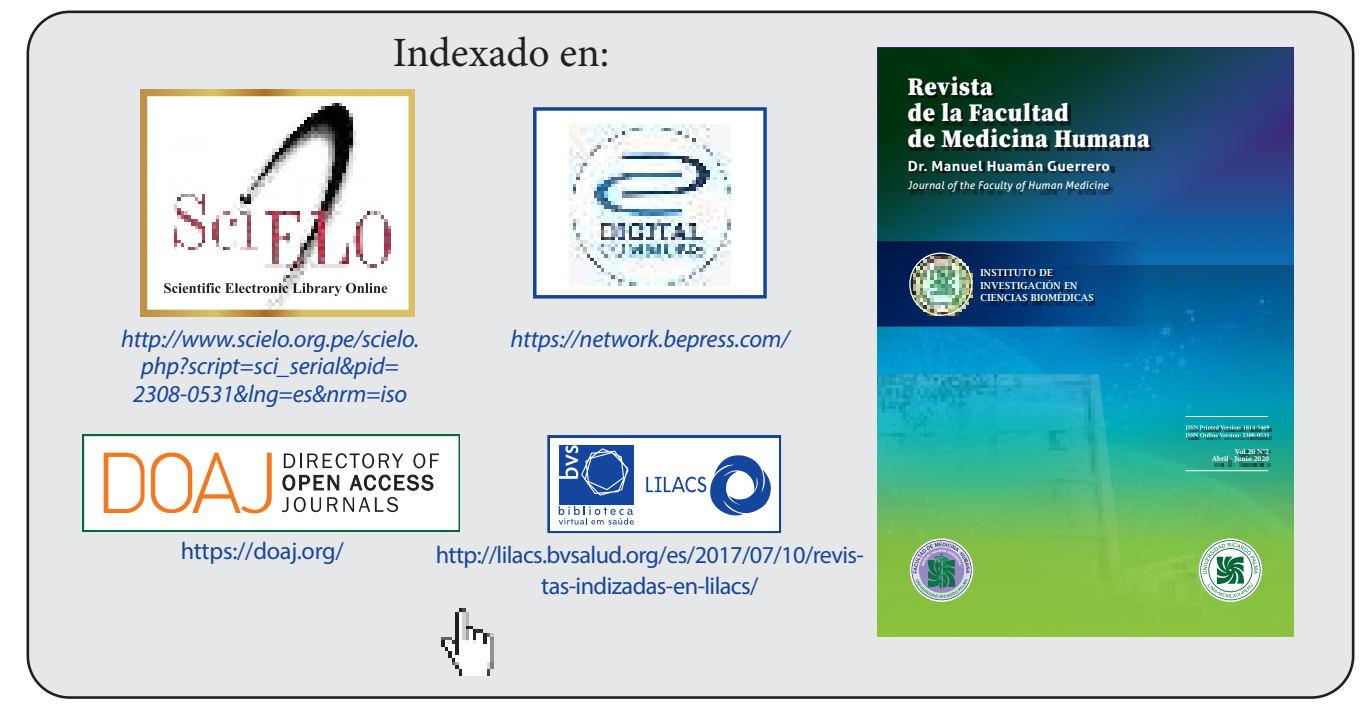

